\title{
The SH2 domain is crucial for function of Fyn in neuronal migration and cortical lamination
}

\author{
Xi Lu, Xinde Hu, Lingzhen Song, Lei An, Minghui Duan, Shulin Chen \& Shanting Zhao \\ College of Veterinary Medicine, Northwest A\&F University, Yangling, Shaanxi 712100, P. R. China
}

\begin{abstract}
Neurons in the developing brain form the cortical plate (CP) in an inside-out manner, in which the late-born neurons are located more superficially than the early-born neurons. Fyn, a member of the Src family kinases, plays an important role in neuronal migration by binding to many substrates. However, the role of the Src-homology 2 (SH2) domain in function of Fyn in neuronal migration remains poorly understood. Here, we demonstrate that the $\mathrm{SH} 2$ domain is essential for the action of Fyn in neuronal migration and cortical lamination. A point mutation in the Fyn SH2 domain $\left(F^{\mathrm{R} 176 \mathrm{~A}}\right)$ impaired neuronal migration and their final location in the cerebral cortex, by inducing neuronal aggregation and branching. Thus, we provide the first evidence of the Fyn $\mathrm{SH} 2$ domain contributing to neuronal migration and neuronal morphogenesis. [BMB Reports 2015; 48(2): 97-102]
\end{abstract}

\section{INTRODUCTION}

The mammalian neocortex is composed of a birthdate-dependent inside-out alignment of six layers of neurons. During corticogenesis, new-born neurons derive from the ventricular zone (VZ)/subventricular zone (SVZ) pass through a multipolar stage to become bipolar and then undergo radial glia-dependent migration to their final destination. The migration of bipolar neurons requires elaborate coordination of leading process extension and somal translocation $(1,2)$. Defects in neuronal migration and cell morphogenesis in the cerebral cortex cause specific neurological syndromes, such as epilepsy, schizophrenia, and Alzheimer's disease (3-5).

Fyn is a non-receptor protein tyrosine kinase that belongs to Src family kinases (SFK) and plays a key role in regulating cell proliferation, differentiation, and cell migration $(6,7)$. The double knockout mouse of Fyn and Src shows a reeler-like

${ }^{*}$ Corresponding author. Tel: +86-151-9145-0784; Fax: +86-02987091032; E-mail: shantingzhao@hotmail.com

http://dx.doi.org/10.5483/BMBRep.2015.48.2.067

Received 17 March 2014, Revised 7 April 2014, Accepted 9 June 2014

Keywords: Fyn, In utero electroporation, Mice, Neuronal migration, $\mathrm{SH} 2$ domain phenotype (8). Fyn is involved in the Reelin-dependent tyrosine phosphorylation of Dab1, which controls the positioning of radially migrating neurons in the cerebral cortex $(9,10)$. Fyn has also been identified as a signal factor in the organization of the cytoskeleton (11). Neuronal migration requires the coordination of dynamics of F-actin, microtubule, and nucleokinesis. However, the underlying molecular mechanism(s) of Fyn controlling neuronal migration remain(s) poorly understood.

Fyn is composed of multiple domains. The short N-terminal region has a unique function and is most divergent among different SFK members. Following the N-terminal is the Src homology 3 (SH3) domain (85-142 aa), which binds to target proteins through sequences containing proline and hydrophobic amino acids (the classic 'PXXP' consensus). The Src homology 2 (SH2) domain (147-237 aa) recognizes the pYEEI consensus and the kinase domain (271-520 aa) is responsible for the enzymatic activity (12-15). The $\mathrm{SH} 2$ domain can bind to phosphotyrosine motifs that play an essential role in Fyn signal transduction.

An intriguing question is whether the $\mathrm{SH} 2$ domain of Fyn is essential for regulating neuronal migration during brain development. Here, we show that the $\mathrm{SH} 2$ domain of Fyn is required for neuronal migration in vivo. $\mathrm{SH} 2$-phosphotyrosine recognition generally involves a highly conserved $\mathrm{SH} 2$ arginine, forming an electrostatic interaction with the phosphate moiety of the ligand (16). Thus, we constructed a point mutation (R176A) in the $\mathrm{SH} 2$ domain. Using in utero electroporation, we first identified that the $\mathrm{Fyn}^{\mathrm{R} 176 \mathrm{~A}}$ mutant showed impaired neuronal migration, in a dose-dependent manner. Furthermore, we observed numerous transfected neurons aggregated in the cortical plate (CP) or intermediate zone (IZ). Finally, we found that the transfected neurons in the CP gave rise to many branches. Thus, our findings indicated that the $\mathrm{SH} 2$ domain of Fyn controlled many aspects of neuronal migration and neuronal morphogenesis.

\section{RESULTS}

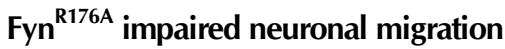

Several studies have reported that Fyn is required for neuronal migration $(8-10,17)$. We hypothesized that the $\mathrm{SH} 2$ domain of Fyn might play an important role in cortical neuronal mig- 
ration. To examine this, we transfected the $\mathrm{Fyn}^{\mathrm{R} 176 \mathrm{~A}}$ plasmid into the newly generated neurons in the neocortex at E15.5 and analyzed 5 days later (at P1), while the pCAG-MCS-GFP plasmid was used as a control. As expected, many transfected cells were mislocated beneath the primitive cortical zone (PCZ), while most of them were located in the PCZ in the control group (Fig. 1). In the Fyn ${ }^{\mathrm{R} 176 \mathrm{~A}}$-transfected group, many neurons were in the $\mathrm{CP}$ and IZ, where very few GFP-positive neurons were found in the control group. Statistical analysis showed that the ratio of neurons in PCZ to CP was significantly different between the two groups. These findings suggested that point mutation of the Fyn $\mathrm{SH} 2$ domain impaired migration and the final location of the cortical neurons.

\section{Fyn $^{\mathrm{R} 176 \mathrm{~A}}$ impaired neuronal migration in a dose-dependent manner}

In the Fyn ${ }^{\text {R176A }}$-transfected group, some neurons also reached the PCZ at P1. Why were only some arrested? We further analyzed the expression of Fyn in different layers, such as the upper CP (UCP), deeper CP (DCP), and IZ. We used GFP as a reporter to indicate the expression of Fyn. To determine average fluorescence intensity of the different layers of the cortex at P1, the fluorescence intensities of GFP-positive cells were measured with ZEN 2011 across a $300 \mu \mathrm{m}$-wide column and normalized with the number of cells. The statistical analysis showed the average fluorescence intensity of the IZ (299.1 \pm 35.69) was significantly higher than the UCP $(116.5 \pm 17.39)$ and DCP $(132.2 \pm 9.238)$. These data indicated that $\mathrm{Fyn}^{\mathrm{R} 176 \mathrm{~A}}$ impaired neuronal migration in a dose-dependent manner. The neurons expressing this mutant at a high level were arrested in the lower layer, while those expressing it at a lower level migrated correctly to the PCZ (Fig. 1). Thus, we demonstrated that Fyn is necessary for neuronal migration, because the $\mathrm{SH} 2$

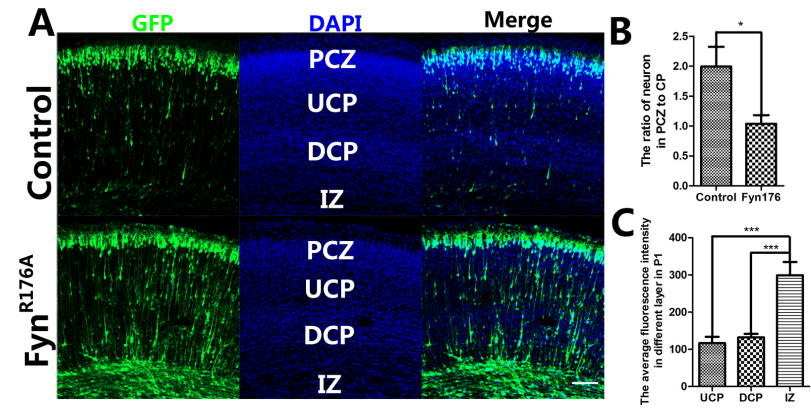

Fig. 1. $F y n^{R 176 A}$ mutant impairs neuronal migration. (A) Coronal sections of P1 brains that had been transfected with GFP plasmid and Fyn ${ }^{\mathrm{R} 176 \mathrm{~A}}$ mutant plasmid at E15.5. The GFP-positive cells showed the transfected cells; many neurons were arrested in the low-CP and $\mathrm{IZ}$ in the $\mathrm{Fyn}{ }^{\mathrm{R} 176 \mathrm{~A}}$ group. (B) the ratio of neurons in PCZ to CP of the two groups (Fyn ${ }^{\text {R1/6A }}$ group compared with GFP control group using the t-test. $* P<0.05)$. (C) Average fluorescent intensity in each layer was analyzed. Neurons in IZ showed significantly stronger fluorescent intensity $(* * * P<0.001)$. Bars indicate mean \pm SEM. (A) Scale bar $=100 \mu \mathrm{m}$. domain mutant resulted in a change in Fyn activity.

\section{Fyn $^{\mathrm{R} 176 \mathrm{~A}}$ induced neuronal aggregation in the $\mathrm{CP}$ and $\mathrm{IZ}$}

Our study showed that overexpression of the $\mathrm{SH} 2$ domain mutant form of Fyn also induced neuronal aggregation in the $\mathrm{CP}$ and IZ of mouse cerebral cortex (Fig. 2). The upper layer neuronal marker 'Brn2' staining suggested that the aggregated neurons in the $\mathrm{CP} / \mathrm{IZ}$ belonged to layer II/III. The aggregation zone was packed densely with cells and clearly demarcated from the surroundings. There was an average of six aggregations in every section. The average cell number in the aggregation zone was $18.6 \pm 4.32$. The average fluorescence intensity of aggregated neurons, normalized to the number of aggregated cells, was $231.1 \pm 14.49$. These results indicated that Fyn could promote neuronal aggregation and adhesion, even when its $\mathrm{SH} 2$ domain was inactive.

\section{Fyn ${ }^{\mathrm{R} 176 \mathrm{~A}}$ promoted the branching of migrating neurons}

As a member of the non-receptor tyrosine kinases, Fyn plays an essential role in actin cytoskeletal organization and cell migration (18-20). The results above show that overexpression of Fyn $^{\mathrm{R} 176 \mathrm{~A}}$ arrested neuronal migration. We hypothesized that Fyn impaired neuronal migration by controlling actin cytoskeleton dynamics and neuronal morphogenesis. To assess this, we examined the morphology of the migrating neurons in the



Fig. 2. Over-expression of $\mathrm{Fyn}^{\mathrm{R} 176 \mathrm{~A}}$ induced neuronal aggregation in the DCP and IZ. (A, B) Sections of P1 brain transfected with Fyn $^{\text {R176A }}$ at E15.5 showed many aggregations formed in the DCP and IZ. $\left(A^{\prime}, B^{\prime}\right)$ Higher magnification of $F y n^{R 176 A}$ positive neurons formed aggregations in the DCP and IZ. The arrow points to the $\mathrm{GFP}^{+} / \mathrm{DAPI}^{+} / \mathrm{Brn}^{+}$(red). (A, B) Scale bar $=100 \mu \mathrm{m},\left(\mathrm{A}^{\prime}\right)$ Scale bar $=20 \mu \mathrm{m},\left(B^{\prime}\right)$ Scale bar $=10 \mu \mathrm{m}$. 
CP. Compared with the GFP control group, striking morphological changes occurred in the neurons transfected with $\mathrm{Fyn}^{\mathrm{R} 176 \mathrm{~A}}$. As shown in Fig. 3 the transfected migrating neurons
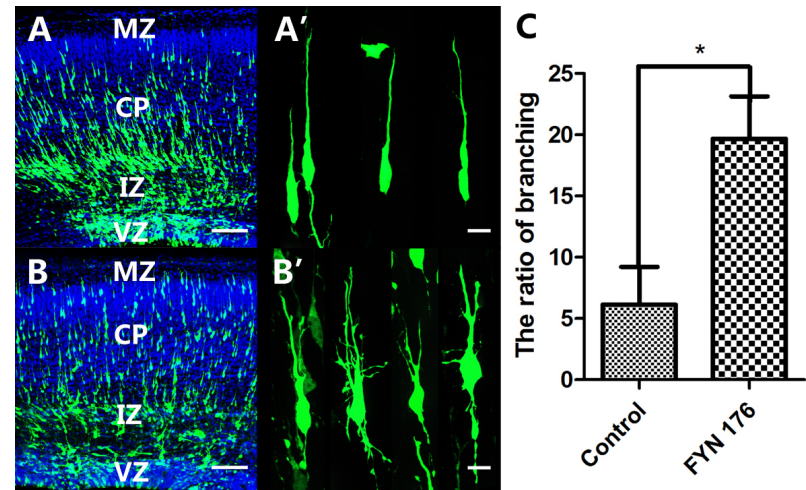

Fig. 3. Expression of $F y n^{\mathrm{R} 176 \mathrm{~A}}$ mutant induced the branching of cortical neurons. The brains were transfected with GFP or Fyn $^{\mathrm{R} 176 \mathrm{~A}}$ at E15.5 and analyzed at E18.5. (A, $\mathrm{A}^{\prime}$ ) Normal morphology of migratory neurons in the GFP control group. $\left(B, B^{\prime}\right)$ Branching of cortical neurons in the Fyn ${ }^{\text {R176A }}$ group. (C) Ratio of neurons with branches in the $\mathrm{Fyn}^{\mathrm{R} 176 \mathrm{~A}}$ mutant group compared with the GFP control group. Bars represent means \pm standard deviation $(* \mathrm{P}<0.05)$. (A, B) Scale bar $=100 \mu \mathrm{m}$, $\left(\mathrm{A}^{\prime}\right.$ and $\left.\mathrm{B}^{\prime}\right)$ Scale bar $=10 \mu \mathrm{m}$. extended many branches and some of them lost the 'classical' neuronal morphology. The ratio of neurons having branches in the Fyn ${ }^{\mathrm{R} 176 \mathrm{~A}}$ expression group reached $19.67 \pm 3.47 \%$, while the GFP control group showed $6.13 \pm 3.08 \%$. Thus, our findings demonstrated that Fyn ${ }^{\mathrm{R} 176 \mathrm{~A}}$ impaired neuronal migration by controlling neuronal morphogenesis.

\section{Fyn ${ }^{\mathrm{R} 176 \mathrm{~A}}$ causes reorganization of F-actin and focal} accumulation of vinculin

We expressed Fyn ${ }^{\mathrm{R} 176 \mathrm{~A}}$ in $\mathrm{CHO}$ cells and stained cytoskeletal components, microtubules and F-actin, as well as the focal adhesion molecule, vinculin. We also transfected 293T cells for Western blot analysis. As shown in Fig. 4G, the expression of Fyn ${ }^{\text {R176A }}$ did not affect the total amount of tubulin, actin, or vinculin. Immunochemistry results showed that Fyn ${ }^{\mathrm{R} 176 \mathrm{~A}}$ induced the depolymerization of F-actin. Well-organized F-actin was detected in the control group, while very little typical $\mathrm{F}$-actin was found in the $\mathrm{Fyn}^{\mathrm{R} 176 \mathrm{~A}}$ group (Fig. 4). Expression of $\mathrm{Fyn}^{\mathrm{R} 176 \mathrm{~A}}$ also induced focal accumulation of the adhesion molecule, vinculin. As shown in Fig. 4, Fyn ${ }^{\text {R176A }}$ partly colocalized with bundled vinculin, while no 'typical' bundled vinculin was observed in the control group (Fig. 4E). The expression and organization of another cytoskeletal component, microtubules, was not affected by the expression of Fyn ${ }^{\text {R176A }}$. Thus, we further demonstrated the mechanism by which Fyn ${ }^{\text {R176A }}$ controls neuronal aggregation, adhesion, and branching.

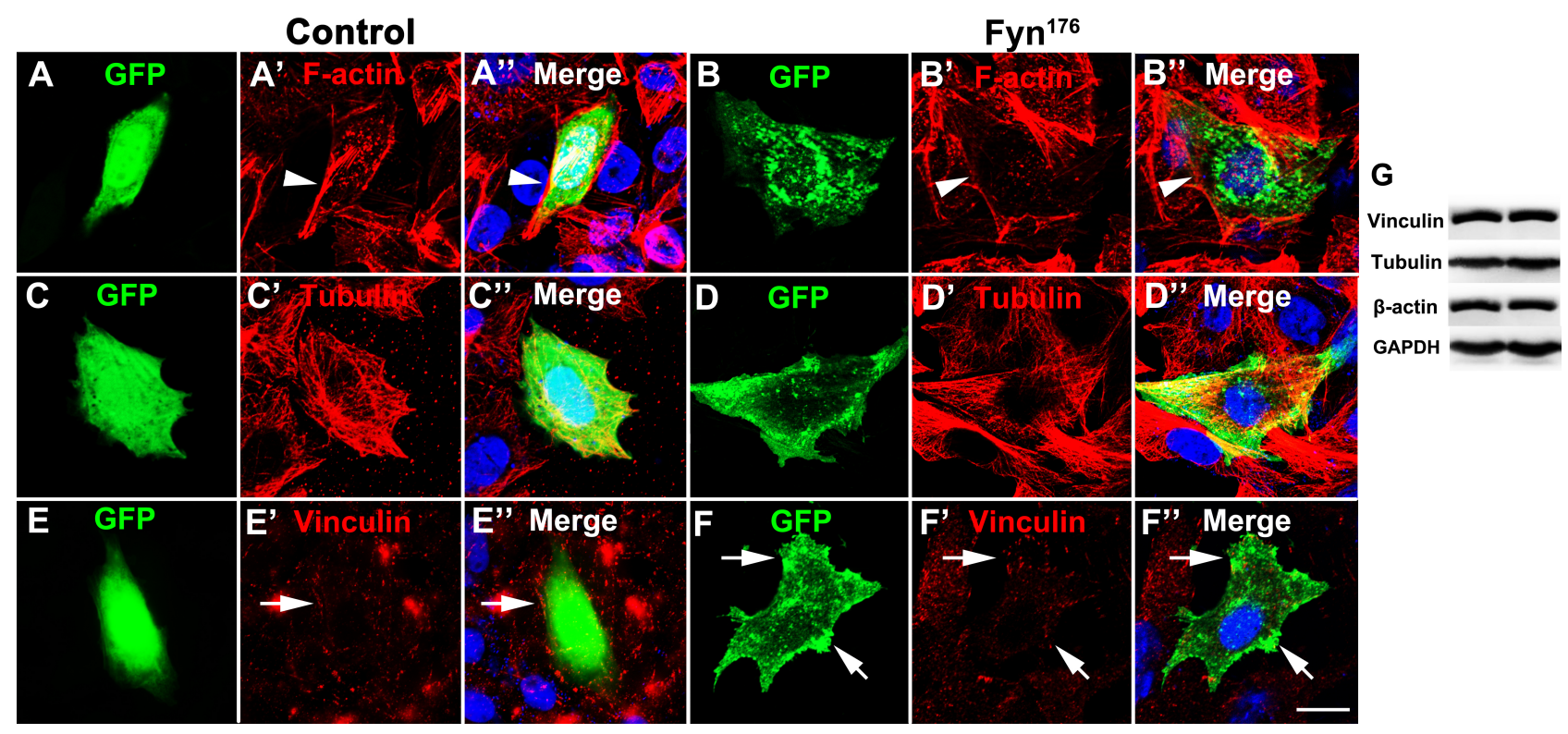

Fig. 4. $F y n^{\mathrm{R} 176 \mathrm{~A}}$ induced reorganization of $\mathrm{F}$-actin and focal accumulation of vinculin $\mathrm{CHO}$ cells were cultured in $\mathrm{F} 12 \mathrm{~K}$ medium containing $10 \%$ FBS and transfected with GFP or Fyn ${ }^{\mathrm{R} 176 \mathrm{~A}}$. (A, B) CHO cells were stained with phalloidin and DAPI, Fyn ${ }^{\mathrm{R} 176 \mathrm{~A}}$ induced the depolymerization of F-actin. (C, D) $\mathrm{CHO}$ cells were stained with tubulin; no difference was observed between the GFP group and Fyn ${ }^{\mathrm{R} 176 \mathrm{~A}}$ group. $(\mathrm{E}, \mathrm{F}) \mathrm{CHO}$ cells were stained with vinculin, and $\mathrm{Fyn}^{\mathrm{R} 176 \mathrm{~A}}$ partially colocalized with vinculin and induced focal accumulation of vinculin. (G) Western blotting analysis of vinculin, tubulin, and actin in the GFP group and Fyn ${ }^{\text {R176A }}$ group; no difference was observed between these two groups. Scale bar $=20 \mu \mathrm{m}$ 


\section{DISCUSSION}

The conserved arginine in the phosphotyrosyl-binding pocket of the $\mathrm{SH} 2$ domain forms essential electrostatic interactions with the bound phosphorylated tyrosine (16). It is typically the case that mutation of this arginine to other residues will greatly weaken the interaction with the ligand but otherwise be tolerated structurally (21). This indicates that a 'negative' mutant may or may not influence the activation of Fyn.

Neuronal migration requires the elaborate coordination of many molecules in multiple steps $(1,22,23)$. Previous studies have shown that Fyn acts as an intracellular signal molecule of Reelin, an extracellular matrix protein known to be essential for brain development (10). By binding to its two receptors, VLDLR and ApoER2, Reelin induces the tyrosine phosphorylation of the cytoplasmic protein Dab1 via SFKs (9). Fyn, a member of the SFKs, binds to the phosphorylated Dab1 with its $\mathrm{SH} 2$ domain to promote Dab1 phosphorylation. Phosphorylated Dab1 transduces the signal to downstream molecules (9). Our results showed that expression of Fyn ${ }^{\text {R176A }}$ disturbed neuronal migration, and many neurons were arrested in the $\mathrm{CP}$ or IZ. As $\mathrm{Fyn}^{\mathrm{R} 176 \mathrm{~A}}$ is the $\mathrm{SH} 2$ domain mutant form, it may disturb the interaction between Fyn and downstream molecules, such as Dab1, Lis1, and FAK. These perhaps contribute to the defects in cortical neuronal migration.

Many Fyn ${ }^{\mathrm{R} 176 \mathrm{~A}}$ overexpressing neurons arrested in the $\mathrm{CP}$ or IZ aggregated to form a big column. Brn2 staining demonstrated that they were later-born neurons, belonging to the upper layers. Previous studies have shown that Fyn interact with many adhesion molecules including integrin $\beta 1$, neural cell adhesion molecule (NCAM), and FAK (20, 24, 25). Fyn is a molecule downstream of NCAM, and is enriched in lipid rafts for the interactions of NCAM with both signaling and cytoskeletal components (26-28). Detailed analyses on the expression level of Fyn ${ }^{\mathrm{R} 176 \mathrm{~A}}$ showed that the aggregated neurons strongly expressed Fyn protein; these neurons were the major group of the migration-defective neurons. These results indicated that Fyn probably promotes neuronal adhesion to control neuronal migration in a dose- and activity-dependent manner. Also, overexpression of the Fyn ${ }^{\mathrm{R} 176 \mathrm{~A}}$ mutant induced the branching of migrating neurons. It is crucial for migrating neurons to keep the dynamics of F-actin organization in the growth cone of the leading process, and Fyn-regulated reorganization of the actin cytoskeleton is probably involved in the process. Fyn regulates F-actin dynamics through Rac1, Cdc42, RhoA, Arp2/3, $\mathrm{N}$-WASP, and Cofilin, which induces the formation of filopodia and lamellipodia (29-31). These indicate the importance of the non-receptor tyrosine kinase Fyn in Reelin-induced neuronal branching in the MZ.

The $\mathrm{SH} 2$ domain has been found to participate in protein-protein interactions involving phosphotyrosine motifs. However, the aggregation and branching suggest that the interaction of Fyn with other proteins was not abolished. The $\mathrm{SH} 2$ domain functions mainly in signal transduction, even though the $\mathrm{SH} 2$-negative form of Fyn still can induce neuronal aggregation and branching. An in vitro analysis also showed that Fyn $^{\text {R176A }}$ can introduce the reorganization of F-actin and the adhesion molecule, vinculin. These results confirmed that inactivation of the $\mathrm{SH} 2$ domain did not completely prevent the activation of Fyn.

In summary, we have shown that the $\mathrm{SH} 2$ domain of Fyn plays an essential role in Reelin signaling transduction, and an $\mathrm{SH} 2$ domain mutant disturbs neuronal migration in a dose-dependent manner. We found that $\mathrm{SH} 2$ domain mutant perturbed neuronal final localizations and induced neuronal aggregation. A detailed analysis revealed that Fyn impaired neuronal morphology. Thus, Fyn regulates neuronal migration by controlling neuronal morphogenesis in a $\mathrm{SH} 2$ domain-dependent manner.

\section{MATERIALS AND METHODS}

\section{Animals}

C57BL/6J mice were housed in our lab. All animals were fed in clean environment with food and water available freely. All animal experiments were performed according to the institutional guidelines for animal experiments.

\section{Plasmid construction}

Fyn coding sequences were cloned from mouse brain cDNA using PCR. The specific primers used were Fyn-wt-forward (5'-CGGAATTCATGGGCTGTGTGCAA-3') and Fyn-wt-reverse (5'-ACGCGTCGACTCACAGGTTTTCACCG-3'); ECOR I and Sal I sites were added to the primers, respectively. We used the pCAG-MCS-Fyn-wt vector as a template to enable preparation of the Fyn mutant using the MutanBEST Kit system (TaKaRa, Japan). The specific primers for Fyn ${ }^{\text {R176A }}$ were $\mathrm{Fyn}^{\mathrm{R} 176 \mathrm{~A}}$-forward (5'-TACATTCTCAGCAATTACG-3'), and Fyn ${ }^{\text {R176A }}$-reverse (5'ACCAGTTTCCCCGGTTGTC-3').

\section{In utero electroporation}

In utero electroporation was performed as previous described (32). Briefly, pregnant mice were anesthetized with sodium pentobarbitone $(50 \mathrm{mg} / \mathrm{g})$, and the uterine horns were exposed. Plasmid DNA at a concentration of $5 \mu \mathrm{g} / \mu \mathrm{l}$, with Fast Green solution $(0.1 \%)$ to monitor the injection, was injected into the embryos' lateral ventricle with a glass micropipette. For electroporation, five pulses separated by $950 \mathrm{~ms}$ were applied at $35 \mathrm{~V}$. The uterine horns were placed back into the abdominal cavity to allow the embryos to continue normal development.

\section{Slice preparation and staining}

At postnatal day 1 (P1) or embryo day 18.5 (E18.5), the brains were fixed with $4 \%$ paraformaldehyde (PFA) for $48 \mathrm{~h}$ at $4^{\circ} \mathrm{C}$. Brains were cut in $50 \mu \mathrm{m}$ sections with a Leica VT $1000 \mathrm{~S}$ vibratome (Leica Microsystems). The tissue sections were incubated with blocking solution (containing $0.1 \mathrm{M} \mathrm{PBS}, 5 \%$ se- 
rum, $1 \% \mathrm{BSA}$ and $0.2 \%$ Triton $\mathrm{X}-100$ ) at RT for $2 \mathrm{~h}$. The sections were incubated with the rabbit-anti-GFP $(1: 1,000$, Millipore) and goat-anti-Brn2 (1: 1,000, Santa Cruz Biotechnology Inc.) overnight at $4^{\circ} \mathrm{C}$. After rinsing in $0.1 \mathrm{M} \mathrm{PBS}$, the sections were incubated with donkey-anti-rabbit 488 (1:300, Millipore) and donkey-anti-goat 555 ( $1: 300$, Millipore) for $3 \mathrm{~h}$ at RT. Then, the sections were counterstained with DAPI for 20 min and rinsed in $0.1 \mathrm{M}$ PBS for $1 \mathrm{~h}$. The sections were mounted in Dako Fluorescent Mounting Medium on glass slides and placed overnight at $4^{\circ} \mathrm{C}$.

\section{Cell culture and immunochemistry}

After transfecting with the plasmid pCAG-MCS-GFP and the $\mathrm{Fyn}^{\mathrm{R} 176 \mathrm{~A}}$ plasmid, $\mathrm{CHO}$ cells were plated on glass coverslips in a $24-$ well dish. When confluency reached $50-60 \%$, the cells were fixed with $4 \%$ paraformaldehyde and immunostained with mouse anti-tubulin ( $1: 1,000$, Millipore), mouse anti-vinculin (1:1,000, Millipore), or TRITC-phalloidin (1:1,000, Millipore) at $4^{\circ} \mathrm{C}$ overnight. The cells were then rinsed and stained with donkey anti-mouse $568(1: 300$, Invitrogen) diluted in $2 \% \mathrm{BSA}$ for $2 \mathrm{~h}$ in the dark, then counterstained with DAPI (1: 500, Invitrogen). Following three additional rinses, coverslips were mounted with Dako fluorescent mounting medium and photographed by a structured-illumination microscope (Zeiss observer Z1).

\section{Immunoblotting}

After transfecting with the plasmid pCAG-MCS-GFP and the $\mathrm{Fyn}^{\mathrm{R} 176 \mathrm{~A}}$ plasmid, soluble 293T cell lysates (equivalent to $5 \times$ $10^{6}$ cells/condition) were subjected to immunoblotting with the following antibodies: mouse anti-vinculin $(1: 1,000$, Millipore), mouse anti- $\beta$-actin ( $1: 2,000$, Sigma), mouse antitubulin $(1: 5,000$, Sigma), and mouse anti-GAPDH $(1: 5,000$, Sigma). Antibody complexes were detected with horseradish peroxidase-conjugated goat anti-mouse immunoglobulin ( $1: 2,000$, Cell signaling Technology) and enhanced chemiluminescence $(E C L)$ reagents.

\section{Statistical analyses}

At least six slices from three brains in separate experiments were analyzed for each group. Image analysis/cell counting was done with the ZEN 2011 program. Statistical differences were analyzed with unpaired t-tests, using GraphPad Prism 5. In all cases, confidence intervals were set at $95 \%$ and $P$ values less than 0.05 were considered significant.

\section{ACKNOWLEDGEMENTS}

This work was supported by Foundation for Special Talent of NWSUAF (No. Z111021101) and The National Natural Science Foundation of China (No. 31071873).

\section{REFERENCES}

1. Ayala R, Shu T and Tsai LH (2007) Trekking across the brain: the journey of neuronal migration. Cell 128, 29-43

2. Nadarajah B, Brunstrom JE, Grutzendler J, Wong RO and Pearlman AL (2001) Two modes of radial migration in early development of the cerebral cortex. Nat Neurosci 4, 143-150

3. Rakic P (2003) Developmental and evolutionary adaptations of cortical radial glia. Cereb Cortex 13, 541-549

4. Valiente M and Marin O (2010) Neuronal migration mechanisms in development and disease. Curr Opin Neurobiol 20, 68-78

5. Marin $\mathrm{O}$ and Rubenstein JL (2003) Cell migration in the forebrain. Annu Rev Neurosci 26, 441-483

6. Brown MT and Cooper JA (1996) Regulation, substrates and functions of src. Biochim Biophys Acta 1287, 121149

7. Klinghoffer RA, Sachsenmaier C, Cooper JA and Soriano P (1999) Src family kinases are required for integrin but not PDGFR signal transduction. EMBO J 18, 2459-2471

8. Kuo G, Arnaud L, Kronstad-O'Brien P and Cooper JA (2005) Absence of Fyn and Src causes a reeler-like phenotype. J Neurosci 25, 8578-8586

9. Arnaud L, Ballif BA, Forster E and Cooper JA (2003) Fyn tyrosine kinase is a critical regulator of disabled-1 during brain development. Curr Biol 13, 9-17

10. Bock HH and Herz J (2003) Reelin activates SRC family tyrosine kinases in neurons. Curr Biol 13, 18-26

11. Xu D, Kishi H, Kawamichi H, Kajiya K, Takada $Y$ and Kobayashi S (2007) Involvement of Fyn tyrosine kinase in actin stress fiber formation in fibroblasts. FEBS Lett 581, 5227-5233

12. Engen JR, Wales TE, Hochrein JM et al (2008) Structure and dynamic regulation of Src-family kinases. Cell Mol Life Sci 65, 3058-3073

13. Boggon TJ and Eck MJ (2004) Structure and regulation of Src family kinases. Oncogene 23, 7918-7927

14. Brickell PM (1992) The p60c-src family of protein-tyrosine kinases: structure, regulation, and function. Crit Rev Oncog 3, 401-446

15. Ingley E (2008) Src family kinases: regulation of their activities, levels and identification of new pathways. Biochim Biophys Acta 1784, 56-65

16. Waksman G, Shoelson SE, Pant N, Cowburn D and Kuriyan J (1993) Binding of a high affinity phosphotyrosyl peptide to the $\mathrm{Src} \mathrm{SH} 2$ domain: crystal structures of the complexed and peptide-free forms. Cell 72, 779-790

17. Forster $E$, Jossin $Y$, Zhao $S$, Chai $X$, Frotscher $M$ and Goffinet AM (2006) Recent progress in understanding the role of Reelin in radial neuronal migration, with specific emphasis on the dentate gyrus. Eur J Neurosci 23, 901-909

18. Martin-Cofreces NB, Sancho D, Fernandez E et al (2006) Role of Fyn in the rearrangement of tubulin cytoskeleton induced through TCR. J Immunol 176, 4201-4207

19. Klein C, Kramer EM, Cardine AM, Schraven B, Brandt R and Trotter J (2002) Process outgrowth of oligodendrocytes is promoted by interaction of Fyn kinase with the cytoskeletal protein Tau. J Neurosci 22, 698-707 
20. Cary LA, Chang JF and Guan JL (1996) Stimulation of cell migration by overexpression of focal adhesion kinase and its association with Src and Fyn. J Cell Sci 109, 1787-1794

21. Zhang Z, Shen K, Lu W and Cole PA (2003) The role of C-terminal tyrosine phosphorylation in the regulation of SHP-1 explored via expressed protein ligation. J Biol Chem 278, 4668-4674

22. Sekine K, Kawauchi T, Kubo K et al (2012) Reelin controls neuronal positioning by promoting cell-matrix adhesion via inside-out activation of integrin alpha5beta1. Neuron 76, 353-369

23. Franco SJ, Martinez-Garay I, Gil-Sanz C, Harkins-Perry SR and Muller U (2011) Reelin regulates cadherin function via Dab1/Rap1 to control neuronal migration and lamination in the neocortex. Neuron 69, 482-497

24. Bodrikov V, Leshchyns'ka I, Sytnyk V, Overvoorde J, den Hertog J and Schachner M (2005) RPTP alpha is essential for NCAM-mediated p59(fyn) activation and neurite elongation. J Cell Biol 168, 127-139

25. Reddy KB, Smith DM and Plow EF (2008) Analysis of Fyn function in hemostasis and alphallbbeta3-integrin signaling. J Cell Sci 121, 1641-1648

26. Santuccione A, Sytnyk V, Leshchyns'ka I and Schachner M (2005) Prion protein recruits its neuronal receptor
NCAM to lipid rafts to activate p59fyn and to enhance neurite outgrowth. J Cell Biol 169, 341-354

27. Ditlevsen DK, Povlsen GK, Berezin V and Bock E (2008) NCAM-induced intracellular signaling revisited. J Neurosci Res 86, 727-743

28. Samayawardhena LA, Kapur R and Craig AW (2007) Involvement of Fyn kinase in Kit and integrin-mediated Rac activation, cytoskeletal reorganization, and chemotaxis of mast cells. Blood 109, 3679-3686

29. Liang X, Draghi NA and Resh MD (2004) Signaling from integrins to Fyn to Rho family GTPases regulates morphologic differentiation of oligodendrocytes. J Neurosci 24, 7140-7149

30. Suetsugu S, Tezuka T, Morimura T et al (2004) Regulation of actin cytoskeleton by mDab1 through N-WASP and ubiquitination of mDab1. Biochem J 384, 1-8

31. Chai X, Forster E, Zhao S, Bock $\mathrm{HH}$ and Frotscher $\mathrm{M}$ (2009) Reelin acts as a stop signal for radially migrating neurons by inducing phosphorylation of n-cofilin at the leading edge. Commun. Integr Biol 2, 375-377

32. Nishimura $Y V$, Shinoda $T$, Inaguma $Y$, Ito $H$ and Nagata $K$ (2012) Application of in utero electroporation and live imaging in the analyses of neuronal migration during mouse brain development. Med Mol Morphol 45, 1-6 